\title{
Exploring controls on the geochemistry of non-spinose planktic foraminifera via culture experiments, plankton tow specimens, and micron- scale analyses.
}

\section{JENNIFER FEHRENBACHER}

Oregon State University

Presenting Author: jennifer.fehrenbacher@oregonstate.edu

Decades ago, geochemical analyses of foraminiferal calcite required large sample sizes (up to 80 foraminifera) that we now commonly refer to as a 'bulk' geochemical analysis. More recently, new analytical tools have given researchers the opportunity to probe the geochemical composition of individual foraminifera at the micron-, submicron- and even atom-scale. Here, we demonstrate how micron-scale analyses (laser ablation, nanoSIMS, and MicroCT imaging) shed light on the mechanisms responsible for trace element variability in non-spinose foraminifera and how we can use these tools to expand their utility in paleoclimate reconstructions.

Exploiting the trace element composition of non-spinose foraminifera for paleoceanographic reconstructions requires an understanding of mechanisms responsible for the incorporation of trace elements into the shell calcite. While temperature appears to be the primary control on the $\mathrm{Mg} / \mathrm{Ca}$ ratio in planktic foraminifera (secondary controls include salinity and $\mathrm{pH}$ ), the incorporation of other trace elements, such as $\mathrm{Ba}$ and $\mathrm{Mn}$, is poorly constrained. These elements are particularly difficult to study in bulk samples because they are also used as diagenetic indicators and, if elevated, researchers have employed corrosive cleaning steps to reduce their concentration.

Using samples from laboratory culture experiments and plankton tows we demonstrate that elements like $\mathrm{Ba}, \mathrm{Mn}$, and $\mathrm{Zn}$ are highly elevated in non-spinose foraminifera species, incorporated during calcification, and often appear 'banded' (alternating high and low trace element 'bands'). These elements are elevated primarily in the early ontogenic calcite and are low and invariable in the outer calcite that forms later ontogeny. We also demonstrate how the trace metal cleaning process, used to prepare samples for analysis, preferentially removes inner calcite (via corrosive cleaning steps or preferential fragmentation of the early calcite) and how dissolution on the seafloor alters shell calcite in a similar manner (early calcite dissolves first). For the non-spinose species, we recommend using individual foraminifera analysis (IFA) for generating geochemical analyses at key intervals of interest rather than solution-based analyses, which are more problematic for the interpretation of these elements. We show preliminary results demonstrating the potential utility of $\mathrm{Ba} / \mathrm{Ca}$ ratios in non-spinose species (measured using the IFA technique) for reconstructing paleoproductivity. 